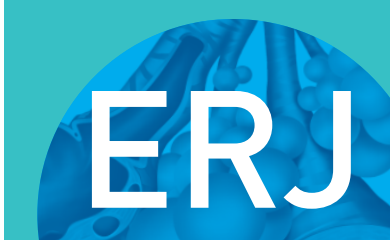

open research

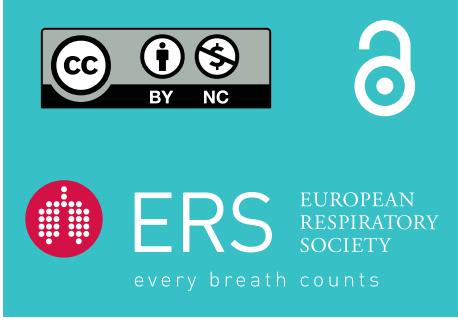

\section{Thrombolysis for massive pulmonary embolisms in morbid obesity: a multisite case-control study}

\section{To the Editor:}

Massive or high-risk pulmonary embolism (PE) confers significant mortality risk and systemic thrombolysis is widely accepted as the first-line treatment in patients without contraindications [1]. High-risk PE in patients with morbid obesity presents unique challenges concerning initial treatments and interventions; in particular, uncertainty over efficacy of weight-based dose adjustments and bleeding complications with antithrombotic therapy [2]. With increasing prevalence of obesity and its independent association with venous thromboembolism (VTE) [3], addressing challenges in management in this patient population has become increasingly relevant. This study evaluated the efficacy and safety of systemic thrombolysis given for massive PE in morbidly obese patients compared to a matched cohort of patients with normal body mass index (BMI).

Patients with acute massive PE admitted to three hospitals in Queensland, Australia (Princess Alexandra Hospital, Logan City Hospital and Sunshine Coast University Hospital) over a 36-month period between June 2016 and June 2019 were screened. All patients who received systemic thrombolysis with a tissue plasminogen activator (tPA) on an intention-to-treat basis for confirmed massive PE on a computed tomographic pulmonary angiogram (CTPA) scan were included. Patients who underwent mechanical clot retrieval (either percutaneous or surgical embolectomy) or had catheter directed thrombolysis were excluded. Massive PE was defined as large clot burden PE with acute haemodynamic instability as characterised by cardiac arrest, obstructive shock or persistent hypotension (systolic blood pressure $<90 \mathrm{mmHg}$ or systolic blood pressure drop $\geqslant 40 \mathrm{mmHg}$ lasting $>15 \mathrm{~min}$ and not caused by new-onset arrhythmia, hypovolaemia or sepsis). The length of follow-up was 12 months.

Outcomes in patients with morbid obesity (weight $>120 \mathrm{~kg}$ or BMI $>40 \mathrm{~kg} \cdot \mathrm{m}^{-2}$ ) were compared to an age-, sex- and pulmonary embolism severity index (PESI) score-matched cohort of nonobese patients. The primary outcome was the rate of all-cause 30-day mortality. Secondary outcomes included: 1) the rate of major and clinically relevant nonmajor bleeding as defined by the International Society of Thrombosis and Haemostasis guidelines [4]; 2) length of hospital stay; 3) rate of re-presentation to hospital within 30 days; 4) rate of recurrent VTE at 6 months; and 5) all-cause mortality at 12 months following the PE.

Estimated frequencies and proportions for the variables were calculated in descriptive analysis. The nonparametric continuous variables were compared using the Mann-Whitney test. The relative risk and 95\% confidence intervals were calculated for rates, and the differences were regarded as significant at a p-value $<0.05$. Ethical approval for this study was granted by the Queensland Metro South Human Research Ethics Committee (HREC/2019/QMS/57882).

A total of 15 morbidly obese patients received upfront systemic thrombolysis during the study period. Two morbidly obese patients who underwent catheter-directed interventions without systemic thrombolysis were excluded. A total of 30 patients (15 in the morbidly obese group and 15 in the matched control group) were included in the study with a median age of 59.7 years (interquartile range $42-73$ years). The mean \pm SD BMI in the 15 patients classified as morbidly obese was $42.7 \pm 6.8 \mathrm{~kg} \cdot \mathrm{m}^{-2}$. All patients had acute

@ERSpublications

This case-control study assessed efficacy and safety of systematic thrombolysis in morbidly obese patients with massive pulmonary embolisms. Thrombolysis at conventional doses seems to have similar efficacy and bleeding rates in morbidly obese patients. https://bit.ly/38ZqJr4

Cite this article as: Samaranayake CB, Keir G, McCabe C, et al. Thrombolysis for massive pulmonary embolisms in morbid obesity: a multisite case-control study. ERJ Open Res 2021; 7: 00762-2020 [https://doi.org/10.1183/23120541.00762-2020].

(c) The authors 2021. This version is distributed under the terms of the Creative Commons Attribution Non-Commercial Licence 4.0. For commercial reproduction rights and permissions contact permissions@ersnet.org 
proximal bilateral PE resulting in right heart strain on diagnostic CTPA. Transthoracic echocardiograms on the day of admission to hospital showed evidence of right ventricular dysfunction and/or raised pulmonary artery systolic pressure in all patients. There was evidence of myocardial damage with elevated cardiac troponin in all patients. The PESI scores were either class IV or V. Baseline clinical characteristics of the two study groups are summarised in table 1.

Patients were treated with either alteplase (loading dose of $10 \mathrm{mg}$ administered intravenously over $2 \mathrm{~min}$ followed by $90 \mathrm{mg}$ over $2 \mathrm{~h}$ for all patients $>65 \mathrm{~kg}$ ) or tenecteplase (a single bolus dose was administered intravenously over $5 \mathrm{~s}$ based on the patients' weight, with a maximum dose of $50 \mathrm{mg}$ ), followed by intravenous heparin infusion for $\geqslant 24 \mathrm{~h}$. All patients were admitted to the intensive care unit for $\geqslant 24 \mathrm{~h}$ following thrombolysis. A total of four patients (two in each group) received half-dose bolus of tPA at the discretion of the treating physician. A minority $(n=3,10 \%)$ did not initially meet the criteria for thrombolysis but due to clinical deterioration in the first $24 \mathrm{~h}$ of the admission, received systemic thrombolysis.

The 30 -day mortality rate was $13.3 \%$ (95\% CI $0.0-32.8 \%$ ) in the obese group and $6.7 \%$ (95\% CI $0.0-20.1 \%)$ in the nonobese group $(\mathrm{p}=0.37)$. Major or clinically relevant nonmajor bleeding was seen in two (13.3\%) obese patients (retroperitoneal and orbital bleeding) and four (26.7\%) nonobese patients (gastrointestinal, retroperitoneal, intercoastal artery and abdominal wall haematoma). The length of hospital stay was higher in the obese group compared to nonobese group (median 11 versus 7 days respectively, $\mathrm{p}=0.04$ ). There was a trend towards an increased rate of re-presentations to hospital within 30 days in the obese group. The reasons for hospital re-presentations were chest pain $(n=3)$, bleeding $(n=1)$, new atrial fibrillation $(n=1)$ and hospital-acquired pneumonia $(n=1)$ in the obese group, and chest pain $(\mathrm{n}=1)$ in nonobese group. The rate of recurrent VTE at 6 months was low ( $\mathrm{n}=0$ in the obese group versus $\mathrm{n}=2$ in the nonobese group). The all-cause mortality within 12 months was similar between the groups $(n=3(20.0 \%, 95 \%$ CI $6.3-45.9 \%)$ in the obese group and $n=2(13.3 \%, 95 \%$ CI $2.5-39.1 \%)$ in the nonobese group). Other than the patients who died, there was no loss to follow-up at 6 months; however, one patient in each group was lost to follow-up at 12 months. Persistent right ventricular dysfunction on transthoracic echocardiography at 6 months was seen in four $(26.7 \%, 95 \%$ CI $10.5-52.4 \%)$ morbidly obese and one $(6.6 \%, 95 \%$ CI $0.0-29.8 \%)$ nonobese patients. The mean right ventricular systolic pressure at 6 months was $29.3 \pm 9.1 \mathrm{mmHg}$ in the morbidly obese group compared to $24.5 \pm 5.5 \mathrm{mmHg}$ in the nonobese group $(p=0.069)$. None of the patients was diagnosed with chronic thromboembolic pulmonary hypertension $(\mathrm{CTEPH})$ during the follow-up period.

This multicentre study found similar 30-day mortality risk for treatment of massive PE with systemic thrombolysis in morbidly obese patients and age-, sex- and PE severity-matched controls. Mortality benefit from thrombolysis in massive PE may be inferred from historic trials [5], although to our knowledge this study is the first study to report outcomes specific to morbidly obese patients. PE-related mortality at 30 days was similar between our study and other real-world data in patients receiving thrombolysis for massive PE [6]. Rates of major bleeding were lower in our study perhaps due to changes in drug distribution in the morbidly obese patient group [2]. Our findings are also consistent with studies in thrombolysis for acute stroke, where obese patients have similar outcomes compared to nonobese patients $[7,8]$.

A strength of this dataset is the high levels of diagnostic work-up including CTPA and echocardiograms substantiating the presence of large clot burdens and acute RV dysfunction. As well as this, treatment decisions were clinician-led within a real-world setting capable of mechanical and circulatory support, increasing the relevance of our findings to centres with PE-specific ICU management pathways. The longer duration of admission in obese patients may be attributed to obese patients receiving more cautionary care, especially given the potential for slower recovery and rehabilitation.

This study has several limitations. The small sample size in each treatment arm prevents the authors from making strong conclusions. The study did not have sufficient power to detect statistically significant differences in rates of bleeding. The clinical utility of half-dose thrombolysis in this patient population cannot be determined due to small patient numbers. Furthermore, the decision to administer systemic thrombolysis can vary between institutions, which may have caused selection bias and impact on the generalisability of our findings. Although none of the patients were diagnosed with CTEPH, routine investigations for chronic thromboembolic disease were at the discretion of individual clinicians and varied somewhat across the sites.

In summary, despite the limitations, the present study shows new and interesting clinical outcome data on thrombolysis of massive PE in real-world morbidly obese patients. Our study provides much needed evidence in support of current thrombolysis protocols in morbidly obese patients with massive PE. Thrombolysis at the conventional doses seems to have similar efficacy and bleeding rates in morbidly obese compared to nonobese patients. The efficacy, safety and feasibility of percutaneous catheter-directed interventions compared to 


\section{TABLE 1 Characteristics of study participants}

\begin{tabular}{|c|c|c|c|}
\hline Characteristics & Morbidly obese ( $n=15$ ) & Nonobese ( $n=15)$ & p-value \\
\hline \multicolumn{4}{|l|}{ Demographics } \\
\hline Age years median (IQR) & $59(42-73)$ & $60(37-75)$ & 0.8 \\
\hline Female & $7(46.7)$ & $7(46.7)$ & \\
\hline \multicolumn{4}{|l|}{ Anthropometrics } \\
\hline Height $\mathrm{cm}$ & $168.9 \pm 9.8$ & $177.3 \pm 5.9$ & 0.02 \\
\hline Weight kg & $129.7 \pm 18.7$ & $86.9 \pm 12.3$ & 0.002 \\
\hline $\mathrm{BMI} \mathrm{kg} \cdot \mathrm{m}^{-2}$ & $42.7 \pm 6.8$ & $27.8 \pm 3.3$ & 0.001 \\
\hline \multicolumn{4}{|l|}{ Pulmonary embolism } \\
\hline \multicolumn{4}{|l|}{ Most proximal clot location } \\
\hline Saddle embolus & 7 (46.7) & 5 (33.3) & 0.39 \\
\hline Main PA & $7(46.7)$ & $10(66.7)$ & 0.28 \\
\hline Lobar arteries & $1(6.7)$ & $0(0)$ & \\
\hline \multicolumn{4}{|l|}{ Clot number } \\
\hline Bilateral & $15(100)$ & $15(100)$ & \\
\hline Right heart strain on CTPA & $15(100)$ & 15 (100) & \\
\hline \multicolumn{4}{|l|}{ Clinical parameters } \\
\hline Cardiac arrest & $1(6.7)$ & $1(6.7)$ & \\
\hline Syncope prior to presentation & 8 (53.3) & $7(46.7)$ & 0.68 \\
\hline First recorded SBP mmHg & $85 \pm 18.8$ & $84 \pm 24.9$ & 0.38 \\
\hline First recorded heart rate beats per min & $110 \pm 19.1$ & $108 \pm 18.1$ & 0.71 \\
\hline $\begin{array}{l}\mathrm{S}_{\mathrm{pO}_{2}}<94 \% \text { on presentation } \\
\text { PESI score }\end{array}$ & $12(80.0)$ & $11(73.3)$ & 0.86 \\
\hline Class V, very high risk & $10(66.7)$ & $10(66.7)$ & \\
\hline Class IV, high risk & $5(33.3)$ & $5(33.3)$ & \\
\hline History of cardiac disease & $1(6.7)$ & $2(13.3)$ & 0.68 \\
\hline History of pulmonary disease & $3(20.0)$ & $1(6.7)$ & 0.61 \\
\hline History of active cancer & $0(0)$ & $1(6.7)$ & \\
\hline \multicolumn{4}{|l|}{ Echocardiographic parameters } \\
\hline LV impairment & 2 (13.3) & $1(6.7)$ & 0.68 \\
\hline \multicolumn{4}{|l|}{ RV impairment } \\
\hline Severe & 4 (26.7) & $4(26.7)$ & \\
\hline Moderate & 5 (33.3) & 2 (13.3) & 0.44 \\
\hline Mild & 8 (53.3) & $3(20.0)$ & 0.22 \\
\hline \multicolumn{4}{|l|}{ RV dilatation } \\
\hline Severe & $6(40.0)$ & $4(26.7)$ & 0.56 \\
\hline Moderate & $1(6.7)$ & $5(33.3)$ & 0.13 \\
\hline Mild & $7(46.7)$ & 5 (33.3) & 0.48 \\
\hline $\mathrm{RVSP}^{\#} \mathrm{mmHg}$ & $47 \pm 12.9$ & $48 \pm 10.1$ & 0.79 \\
\hline \multicolumn{4}{|l|}{ Laboratory markers } \\
\hline Elevated troponin & $15(100)$ & $15(100)$ & \\
\hline Lactate on presentation $\mathrm{mmol} \cdot \mathrm{L}^{-1}$ & $3.9 \pm 2.7$ & $5.1 \pm 3.1$ & 0.25 \\
\hline \multicolumn{4}{|l|}{ Thrombolysis } \\
\hline \multicolumn{4}{|l|}{ Agent } \\
\hline Alteplase & 14 (93.3) & $12(80.0)$ & 0.43 \\
\hline Tenecteplase & $1(6.7)$ & $3(20.0)$ & 0.61 \\
\hline Half dose lysis & 2 (13.3) & $2(13.3)$ & \\
\hline Rescue lysis due to deterioration & 2 (13.3) & $1(6.7)$ & 0.47 \\
\hline \multicolumn{4}{|l|}{ Anticoagulation on discharge } \\
\hline Novel oral anticoagulant & $9(60.0)$ & 8 (53.3) & 0.79 \\
\hline Warfarin & $6(40.0)$ & $6(40.0)$ & \\
\hline Low molecular weight heparin & $0(0.0)$ & $1(6.7)$ & 0.69 \\
\hline
\end{tabular}

Data are presented as $\mathrm{n}(\%)$ or mean \pm SD, unless otherwise stated. IQR: interquartile range; BMI: body mass index; PA: pulmonary artery; CTPA: computed tomography pulmonary angiogram; SBP: systolic blood pressure; $\mathrm{S}_{\mathrm{pO}_{2}}$ : oxygen saturation measured by pulse oximetry; PESI: pulmonary embolism severity index; LV: left ventricle; RV: right ventricle, RVSP: right ventricular systolic pressure. \#: in patients who had a sufficient tricuspid regurgitation velocity measurement.

systemic thrombolysis therapy in morbidly obese patients with acute massive PE remains to be addressed. Larger randomised clinical trials comparing systemic thrombolysis and localised mechanical intervention are needed to determine the optimal treatment strategy for massive PE in this patient population. 
Chinthaka B. Samaranayake $\oplus^{1}$, Gregory Keir ${ }^{1,2}$, Colm McCabe ${ }^{3,4}$, James Anderson ${ }^{5,6}$, Khoa Tran ${ }^{1,7}$ and John W. Upham ${ }^{1,2}$

${ }^{1}$ Faculty of Medicine, University of Queensland, Brisbane, Australia. ${ }^{2}$ Princess Alexandra Hospital, Brisbane, Australia. ${ }^{3}$ Royal Brompton \& Harefield National Health Service Trust, London, UK. ${ }^{4}$ National Heart and Lung Institute, Imperial College, London, UK. ${ }^{5}$ Sunshine Coast University Hospital, Birtinya, Australia. ${ }^{6}$ School of Medicine, Griffith University, Southport, Australia. ${ }^{7}$ Logan Hospital, Brisbane, Australia.

Correspondence: Chinthaka B. Samaranayake, Translational Research Institute, 37 Kent Street, Woolloongabba, Brisbane, Qld 4102, Australia. E-mail: c.samaranayake@uq.edu.au

Received: 19 Oct 2020 | Accepted: 12 Jan 2021

Acknowledgements: The authors would like to thank Mr Roney Neal from the Vascular Medicine Dept at Princess Alexandra Hospital for maintaining the Venous Thromboembolism Database.

Conflict of interest: None declared.

\section{References}

1 Konstantinides SV, Meyer G, Becattini C, et al. 2019 ESC Guidelines for the diagnosis and management of acute pulmonary embolism developed in collaboration with the European Respiratory Society (ERS): The Task Force for the diagnosis and management of acute pulmonary embolism of the European Society of Cardiology (ESC). Eur Respir J 2019; 54: 1901647.

2 Rocca B, Fox KAA, Ajjan RA, et al. Antithrombotic therapy and body mass: an expert position paper of the ESC Working Group on Thrombosis. Eur Heart J 2018; 39: 1672-1686.

3 Movahed MR, Khoubyari R, Hashemzadeh M, et al. Obesity is strongly and independently associated with a higher prevalence of pulmonary embolism. Respir Investig 2019; 57: 376-379.

4 Schulman S, Kearon C. Definition of major bleeding in clinical investigations of antihemostatic medicinal products in non-surgical patients. J Thromb Haemost 2005; 3: 692-694.

5 Marti C, John G, Konstantinides S, et al. Systemic thrombolytic therapy for acute pulmonary embolism: a systematic review and meta-analysis. Eur Heart J 2015; 36: 605-614.

6 Nishimoto Y, Yamashita Y, Morimoto T, et al. Thrombolysis with tissue plasminogen activator in patients with acute pulmonary embolisms in the real world: from the COMMAND VTE registry. J Thromb Thrombolysis 2019; 48: 587-595.

7 Seet RC, Zhang Y, Wijdicks EF, et al. Thrombolysis outcomes among obese and overweight stroke patients: an ageand National Institutes of Health Stroke Scale-matched comparison. J Stroke Cerebrovasc Dis 2014; 23: 1-6.

8 Branscheidt M, Schneider J, Michel P, et al. No impact of body mass index on outcome in stroke patients treated with IV thrombolysis BMI and IV thrombolysis outcome. PLoS One 2016; 11: e0164413. 